\title{
Percutaneous Management of Lymphoceles after Renal Transplantation
}

\author{
Matthew G. Gipson, MD ${ }^{1}$ \\ ${ }^{1}$ Department of Radiology, University of Colorado, Denver Anschutz \\ Medical Campus, Aurora, Colorado \\ Semin Intervent Radiol 2013;30:215-218
}

Address for correspondence Matthew G. Gipson, MD, Stop C278, Research Complex 2, 12700 E. 19th Ave., Room P15-1214, Aurora, CO 80045 (e-mail: matthew.gipson@ucdenver.edu).
Chronic kidney disease (CKD) is a worldwide public health problem. In the United States, between 1988-1994 and 2005-2010, the overall prevalence estimate for CKD, defined by an estimation of glomerular filtration rate $<60 \mathrm{ml} / \mathrm{min}$ per $1.73 \mathrm{~m}^{2}$ or a urine albumin-to-creatinine ratio $\geq 30 \mathrm{mg} / \mathrm{g}$, rose from 12.3 to $14.0 \%$. In 2010, overall per person per year costs for patients with CKD reached \$22,323 for Medicare patients $\geq 65$ years and older, and the overall Medicare expenditure for CKD was $\$ 41.0$ billion, which consisted of $17 \%$ of the total Medicare dollars allocated. ${ }^{1}$

Kidney transplantation is the treatment of choice for patients with end-stage renal disease and should be discussed with patients with advanced CKD preparing for renal replacement therapy. ${ }^{2}$ The annual number of kidney transplants in the United States extends beyond 17,000 patients (deceased donors to living donors), and potential candidates should be referred promptly to a transplant center. Graft dysfunction arising after renal transplant can be divided into urologic (urine leaks or obstruction), vascular, perirenal fluid collections, and infectious complications. Perirenal transplant fluid collections can develop in the immediate postoperative period or over several months. The differential diagnosis of a perirenal fluid collection immediately after surgery includes seroma, hematoma, and urinoma, and fluid collections that develop in a subacute fashion include abscess or lymphoceles.

Lymphoceles are a common and well-documented complication in renal transplant recipients, occurring in up to $26 \%$ of patients. $^{3}$ Usually they are found incidentally on routine ultrasonography and require no additional management; however, a small number are symptomatic due to mass effect and require further definitive treatment. Treatment options include laparoscopic/open surgical or percutaneous therapies with each having different profiles of efficacy and complications. At my institution, we commonly use less invasive percutaneous therapies initially, with surgical management reserved for percutaneous treatment failure. Potential current options for the interventional radiologist include simple aspiration, catheter drainage, and sclerotherapy. In a recent systematic review of 52 retrospective case series (1113 cases of primary lymphocele), treatment efficacy was evaluated and demonstrated recurrence rates of 59\% (141 cases; $n=218$ ), $50 \%$ (100 cases; $n=195$ ), and $31 \%$ (41 cases; $n=155$ ) with aspiration, catheter drainage, and sclerotherapy, respectively. ${ }^{4}$

\section{Patient Selection}

Given the high recurrence rates with current available percutaneous therapies, risk of complications from laparoscopic/ open surgical treatment modalities and need for hospitalization with surgical management, we have recently used an alternative percutaneous strategy in the management of postrenal transplant lymphoceles. This technique involves ultrasound and fluoroscopic-guided percutaneous transperitoneal balloon fenestration of the lymphocele. No specific guidelines have been adopted for patient selection; however, it is usually performed early in the work-up and diagnosis of the lymphocele, and theoretically the procedure is more efficacious with simple unilocular fluid collections. This procedure can also be performed in angiography suites with standard equipment and potentially reduces the dwell time for drainage catheters. More traditional treatment modalities should be entertained if the perirenal lymphocele recurs or resists percutaneous balloon fenestration.

\section{Procedure}

All available imaging modalities should be reviewed before any intervention on a posttransplant fluid collection to assist the operator in treatment. The diagnosis of a lymphocele is usually suspected by ultrasound; however, the first step in treatment of these collections is confirming the diagnosis with aspiration and biochemical analysis of the fluid.

Under moderate sedation, focused ultrasound of the graft is performed in the angiography suite under standard sterile technique to determine the relationship of the fluid collection in respect to the graft, urinary bladder, and adjacent vascular structures. Under direct ultrasound guidance, access the collection with either a $5 \mathrm{~F}$ Yueh centesis catheter needle
Issue Theme Pulmonary Malignancies; Guest Editors, Bradley B. Pua, MD and David C. Madoff, MD, FSIR
Copyright (c) 2013 by Thieme Medical Publishers, Inc., 333 Seventh Avenue, New York, NY 10001, USA. Tel: +1(212) 584-4662.
DOI http://dx.doi.org/ 10.1055/s-0033-1342964. ISSN 0739-9529. 
(Cook Medical, Bloomington, IN) or an 18-gauge needle with Chiba tip, and aspirate $\sim 5 \mathrm{~mL}$ of fluid for biochemical analysis. Submit the fluid to the laboratory for creatinine level, routine culture/gram stain, and cell count with differential. A lymphocele is diagnosed when the creatinine of the aspirated fluid is similar to that of serum and when the specimen contains predominantly lymphocytes. Culture results are useful to diagnosis concomitant infection that may affect the algorithm or timing of treatment.

Place a small-bore multipurpose pigtail drainage catheter into the perigraft fluid collection using standard Seldinger technique and fluoroscopic guidance. A small pigtail catheter that is ethanol compatible such as the 8.5F Dawson-Mueller drainage catheter (Cook Medical, Bloomington, IN) is preferred. This type of catheter gives you flexibility, permitting sclerotherapy with ethanol to treat the fluid collection without the need for a catheter exchange. Connect the catheter to a Merit drainage depot bag (Merit Medical, South Jordan, UT) left open to gravity drainage. The patient is instructed how to care for the drainage catheter, asked to record daily outputs, and scheduled for follow-up outpatient percutaneous therapy within 24 to 48 hours. This gives you time to obtain the necessary laboratory studies, which will confirm the diagnosis, and to acquire a sense of the fluid collection outputs.

Once the diagnosis of a lymphocele is confirmed and after discussion with the referring surgeon, proceed with imageguided percutaneous transperitoneal balloon fenestration. This technique is performed on an outpatient basis under moderate sedation. The indwelling drainage catheter and surrounding abdominal wall are sterilized in standard fashion, and subsequently iodinated contrast (diluted with normal saline) is injected into the lymphocele through the indwelling percutaneous catheter. It is helpful if the patient does not void prior to the examination, allowing direct ultrasound visualization and delineation of the lymphocele with respect to the bladder (-Fig. 1).

Place an 18-gauge needle with Chiba tip into the lymphocele from a midline or paramedian approach, puncturing the
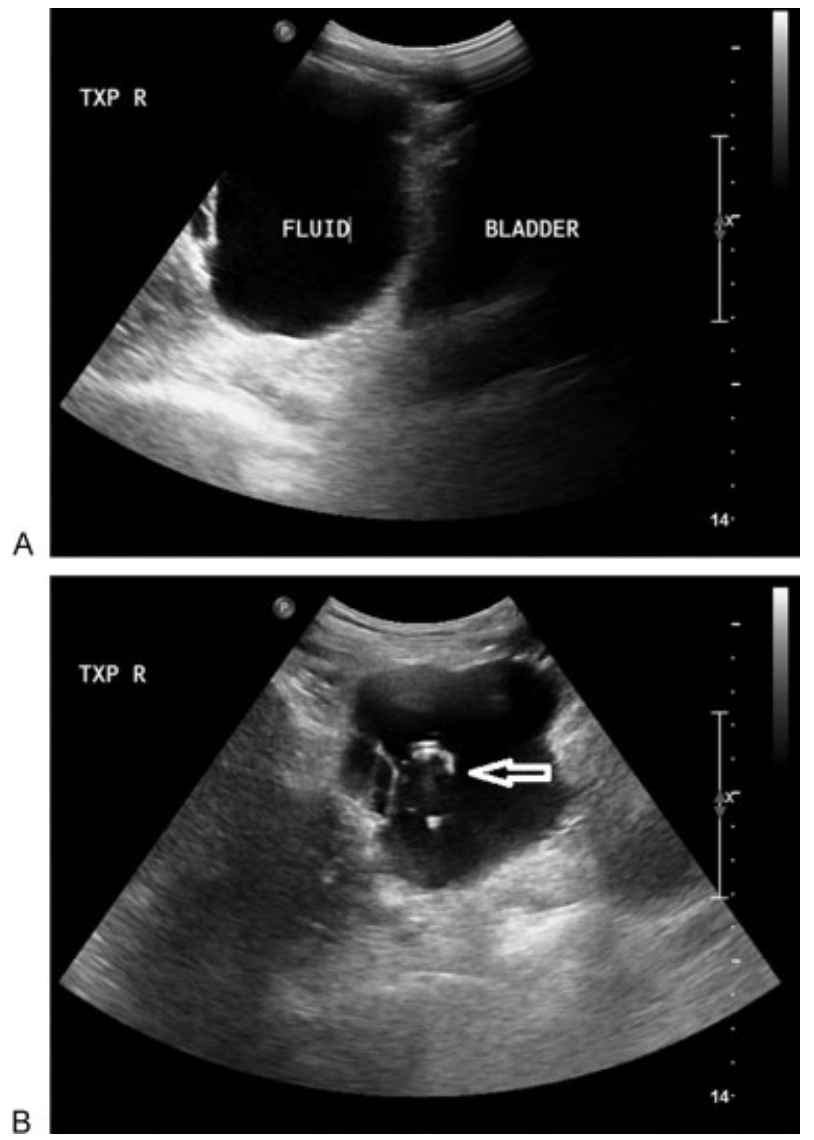

Figure 1 (A) Gray scale ultrasound image demonstrates the relationship of perinephric fluid collection and urinary bladder in the right hemipelvis. (B) The echogenic indwelling small-bore pigtail catheter (arrow) within the center of lymphocele.

collection along its ventral surface. Using the Seldinger technique, advance a 0.035 Bentson guidewire (Cook Medical, Bloomington, IN) under fluoroscopic guidance coiled into the fluid collection (-Fig. 2). Directional access into the fluid collection at this point is important; the goal is to traverse the intraperitoneal space with your wire. As discussed later, this
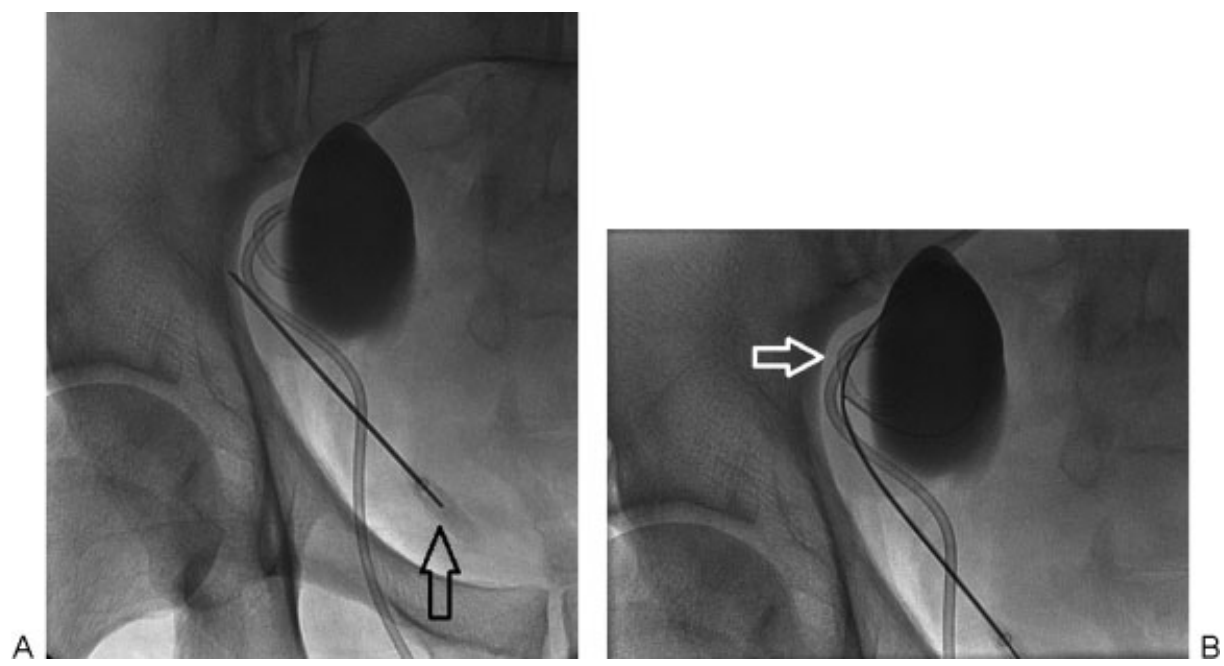

Figure 2 (A) Fluoroscopic spot image demonstrates an 18-gauge Chiba tip needle (arrow) centered in the lymphocele cavity, placed from an anteromedial and ventral approach. (B) A Bentson wire is positioned and coiled into the fluid collection. White arrow shows the small-bore pigtail catheter placed previously into the fluid collection for drainage. 


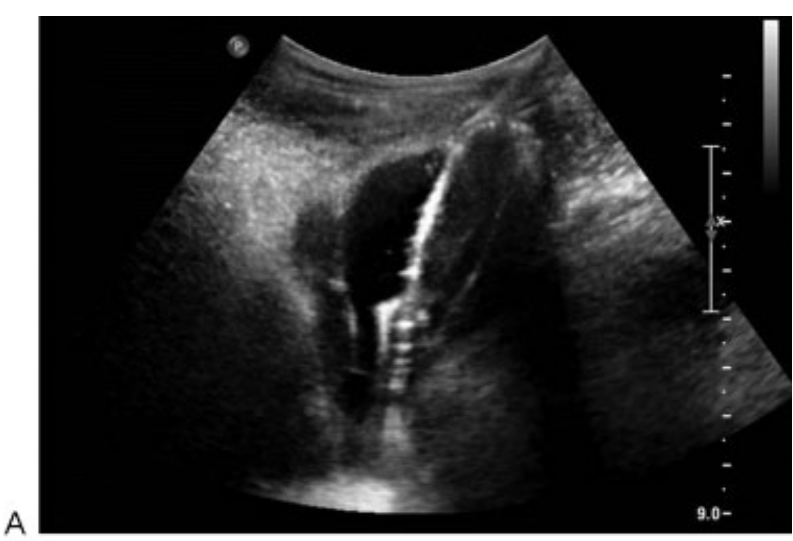

A

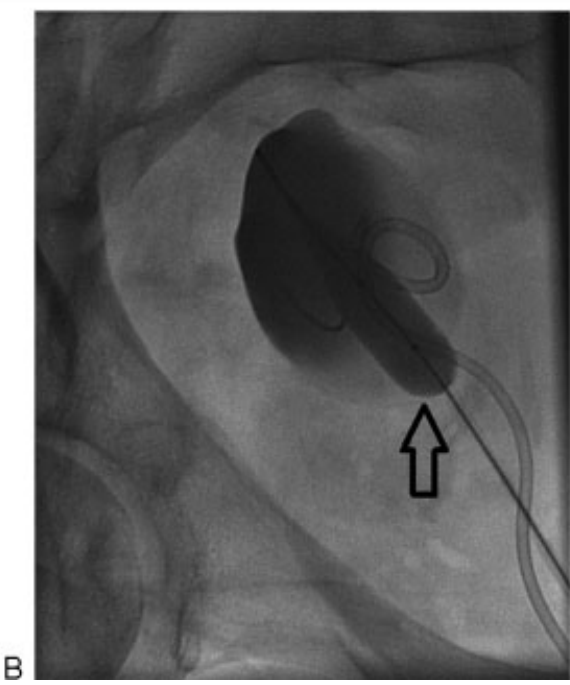

Figure 3 (A) Gray scale ultrasound image and (B) fluoroscopic spot image taken during inflation of a 14-mm angioplasty balloon (arrow) across the ventral wall of the lymphocele over a guidewire.

technique ultimately fenestrates the lymphocele with your angioplasty balloon, thus creating a "window" that communicates with the intraperitoneal space. Careful ultrasound access will allow you to avoid adjacent bowel and vascular structures.

Over wire, I dilate the subcutaneous tissues and place a standard 8F vascular sheath (Cordis Corp., Bridgewater, NJ) with distal tip in the collection. Use a sheath to introduce the angioplasty balloon, which theoretically decreases the risk of rectus muscle trauma that could occur with a "bare-back" balloon over-wire approach. Start with a $14 \mathrm{~F}$ Conquest PTA balloon (Bard Peripheral Vascular, Inc., Tempe, AZ) and advance the device through the sheath. The location of the balloon, with the distal portion in the fluid collection and proximal portion outside the ventral wall of the fluid collection, should be confirmed by fluoroscopy and ultrasound prior to high-pressure inflation. Retract the sheath to allow full expansion of the device; insufflate the balloon for $60 \mathrm{sec}-$ onds and subsequently remove it over wire through the sheath (-Fig. 3).

To evaluate for creation of a fenestration, inject the indwelling small-bore drainage catheter with full-strength contrast, and using digital subtraction angiography (DSA), assess for intraperitoneal extravasation of contrast (-Fig. 4).

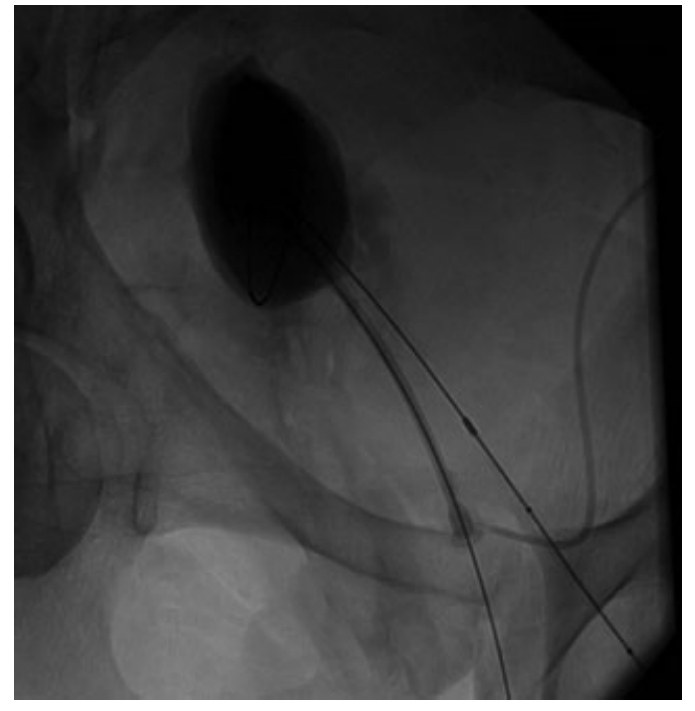

Figure 4 Fluoroscopic spot image obtained after transperitoneal balloon fenestration and balloon removal. Contrast is seen collecting outside of the lymphocele in an inferomedial location. The small-bore pigtail catheter was removed over a wire and balloon fenestration performed through this access (second inflation).

Perform this sequence of events two to three additional times to create multiple fenestrations along the ventral wall of the lymphocele. More fenestrations will optimize drainage, so at this point either reaccess the lymphocele from a different direction or remove the small-bore drainage catheter over wire. Simply upsizing the angioplasty balloon will widen the fenestration and potentially augment drainage. At the end of the procedure, remove the wires and sheaths, and cap the small-bore drainage catheter after aspirating all contents.

Typically the patient is scheduled to return to the interventional radiology department in 48 to 72 hours for ultrasound to assess the need for further treatment. If the lymphocele is not visualized at that time, cut and remove the drain (-Fig. 5). If the lymphocele remains, initiate a second trial of percutaneous balloon fenestration.

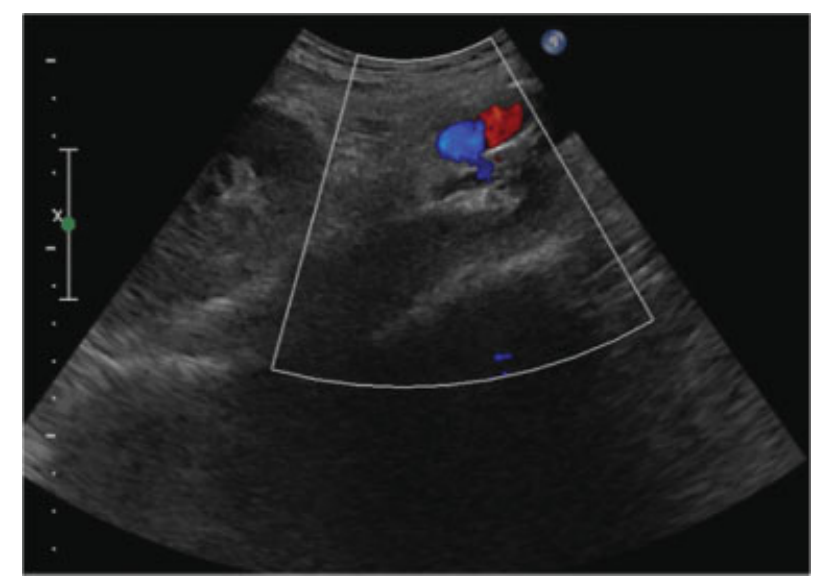

Figure 5 Ultrasound image obtained 2 days after the procedure demonstrates resolution of the lymphocele; the indwelling small-bore drain had been capped for 48 hours. 


\section{Discussion}

CKD is a worldwide public health problem, and the prevalence in the United States has been increasing over the last 2 decades. Kidney transplantation is the treatment of choice for patients with end-stage renal disease with $>17,000$ renal transplants performed in the United States alone. Interventional radiologists are consulted to manage assorted renal transplant complications including perirenal fluid collections. Lymphoceles are a common and well-documented complication in renal transplant recipients with various treatment modalities available including aspiration and drainage (with or without sclerotherapy) and open surgery. We have found that optimal treatment of these patients requires collaboration with the referring transplant surgical team. At my institution we have adopted an alternative percutaneous method to treat lymphoceles with image-guided percutaneous transperitoneal balloon fenestration.

\section{References}

1 United States Renal Data System. USRDS 2012 Annual Data Report: Atlas of Chronic Kidney Disease and End-Stage Renal Disease in the United States. Bethesda, MD: National Institutes of Health, National Institute of Diabetes and Digestive and Kidney Diseases; 2012. Available at:www.usrds.org. Accessed on March 28, 2013

2 Bunnapradist S, Danovitch GM. Evaluation of adult kidney transplant candidates. Am J Kidney Dis 2007;50(5):890-898

3 Atray NK, Moore F, Zaman F, et al. Post transplant lymphocele: a single centre experience. Clin Transplant 2004;18(Suppl 12):46-49

4 Lucewicz A, Wong G, Lam VW, et al. Management of primary symptomatic lymphocele after kidney transplantation: a systematic review. Transplantation 2011;92(6):663-673 